\title{
Histidine-Rich Glycoprotein and Stanniocalcin-2 High Affinity Interactions with Inflammatory Cells
}

Lena Claesson-Welsh ( $\sim$ lena.welsh@igp.uu.se)

Uppsala University

Tor Persson Skare

Uppsala University

Hiroshi Kaito

Uppsala University

Claudia Durall

Attana (Sweden)

Teodor Aastrup

Attana (Sweden)

Research Article

Keywords:

Posted Date: January 17th, 2022

DOI: https://doi.org/10.21203/rs.3.rs-1231761/v1

License: (c) (1) This work is licensed under a Creative Commons Attribution 4.0 International License.

Read Full License 
1

2

3

4

5

6

7

8

9

\section{列}

Histidine-rich glycoprotein and stanniocalcin-2 high affinity interactions with inflammatory cells (1)

Tor Persson Skare ${ }^{1)}$, Hiroshi Kaito ${ }^{1) \S}$, Claudia Durall ${ }^{2)}$, Teodor Aastrup ${ }^{2)}$, Lena Claesson-Welsh ${ }^{1,{ }^{*}}$ (1)

1) Uppsala University, Department of Immunology, Genetics and Pathology, Science for Life and Beijer Laboratories, Dag Hammarskjöldsv 20, 75185 Uppsala, Sweden

2) Attana $A B$, Greta Arwidssons väg 21, 11419 Stockholm, Sweden

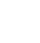

*) to whom correspondence should be directed at the address above or email lena.welsh@igp.uu.se §) Present affiliation: Department of Nephrology, Hyogo Prefectural Kobe Children's Hospital, Kobe, Japan

3

6




\section{Abstract}

The plasma protein Histidine-rich glycoprotein (HRG), is implicated in macrophage polarization to an M1 antitumoral phenotype. The broadly expressed, secreted protein Stanniocalcin 2 (STC2), also implicated in tumor inflammation, is an HRG interaction partner. With the aim to biochemically characterize the HRG and STC2 complex, binding of recombinant HRG and STC2 preparations to each other and to cells was explored using quartz crystal microbalance (QCM) methodology. Protein functionality was tested in a phagocytosis assay, where HRG increased phagocytosis by monocytic U937 cells while STC2 suppressed HRG-induced phagocytosis. Binding of HRG to STC2 measured using QCM showed an affinity between the proteins in the nanomolar range, which occurred in a conformation-dependent manner. Both HRG and STC2 bound individually and in combination to vitamin D3-treated, differentiated U937 monocytes. HRG, but not STC2, also bound to formaldehydefixed U937 cells irrespective of their differentiation stage, involving both a high affinity interaction and binding to heparan sulfate. These data show that binding of HRG to STC2 occurs with high affinity and that HRG and STC2 bind to separate sites on U937 monocytes, suggesting that they exert their effects through distinct cell surface entities. 政

(1) 


\section{Introduction}

Histidine-rich glycoprotein (HRG) is a $75 \mathrm{kDa}$ plasma protein produced by hepatocytes and implicated in cancer immune responsiveness ${ }^{1}$. HRG is organized in a multi-domain structure consisting of two Nterminal cystatin-like domains, followed by a histidine-proline-rich (His/Pro-rich) domain containing 12 pentapeptide repeats of Gly-His-His-Pro-His. The His/Pro repeats, which are highly conserved among mammalian species ${ }^{2}$, are flanked by two Pro-rich regions and a C-terminal domain ${ }^{3}$. The cystatin domains have been implicated HRG's antibacterial effects ${ }^{4}$ and in IgG and complement C1q binding ${ }^{5}$. The His/Pro-rich domain binds heparan sulfate in a $\mathrm{Zn}^{2+}$ dependent manner ${ }^{6}$. This domain is also critical for the anti-angiogenic properties of $\mathrm{HRG}^{7}$.

HRG's multi-domain structure allows interactions with a range of proteins, both intracellular such as tropomyosin, extracellular such as stanniocalcin 2 (STC2), and proteins participating in the coagulation cascade, including plasminogen, plasmin and fibrinogen ${ }^{8,9}$. Consequently, HRG is involved in diverse processes including defence against bacterial infections, in regulation of coagulation and fibrinolysis, inflammation and angiogenesis. Thus, HRG exerts antibacterial effects and may serve as a clinical biomarker for sepsis ${ }^{10}$. Moreover, HRG accelerates both coagulation and fibrinolysis in a $\mathrm{Hrg}^{-/}$mouse model ${ }^{11}$. Rare familiar cases of HRG deficiency supports a role for HRG in regulation of coagulation ${ }^{12}$. Certain HRG effects are dependent on changes in gene regulation in monocytes/macrophages, promoting a phenotypic switch towards anti-tumor immunity and dampened tumor growth and metastasis ${ }^{1}$. While HRG administration to tumor-bearing wildtype mice results in suppressed tumor growth and metastasis, tumor growth is accelerated in $\mathrm{Hrg}^{-/}$mice, and tumor macrophages are predominantly of an $\mathrm{M} 2$ phenotype in the absence of $\mathrm{HRG}^{1,7,13}$.

We have previously investigated the immunomodulatory role of HRG on inflammatory cells, and found that HRG appears in complex with STC2 and that HRG suppresses STC2-mediated gene regulation on U937 monocytic cells ${ }^{14}$. STC2 is a glycosylated homodimeric protein expressed in the placenta, in endothelial cells, fibroblasts and cardiomyocytes ${ }^{15}$. In mouse glioma, tumor-infiltrating leukocytes express STC2 ${ }^{14}$. Like HRG, STC2 is involved in inflammatory processes and in $\mathrm{Ca}^{2+}$ and $\mathrm{PO}_{4}$ homeostasis ${ }^{16,17}$. Stc2 ${ }^{-/}$mice show decreased overall growth, suggesting an important role for STC2 in muscle and bone development ${ }^{18}$.

Here, we first determined the effects of HRG and STC2 recombinant protein preparations in regulation of phagocytosis by U937 monocytes, in order to define the functionality of the recombinant proteins. U937 cells is a human histiocytic lymphoma cell line, capable of differentiating towards a macrophagelike phenotype after treatment with vitamin $D 3^{19}$. While HRG administration stimulated phagocytosis, STC2 abrogated this HRG-dependent effect. To explore the biochemical properties of HRG, STC2 and 
89 the complex, we utilized a novel quartz crystal microbalance (QCM) technique to measure the affinity 90 and characterize the kinetics of the HRG-STC2 interaction as well as the binding of HRG and STC to the 91 monocyte cell surface ${ }^{20}$. We found that only differentiated U937 cells could bind the two proteins, with 92 an affinity in the nanomolar range. Moreover, the protein-protein interaction was highly conformation 93 dependent. These findings confirm the specificity of the HRG-STC2 interaction, and suggest that these 94 proteins have potential as future drug targets for modulation of inflammation. 


\section{Results}

\section{HRG increases phagocytosis by U937 monocytes}

First, the bioactive properties of purified, recombinant STC2 and HRG preparations were determined. To ensure that the purified, recombinant proteins could form a complex as previously shown by coimmunoprecipitation from co-expressing cells ${ }^{14}$, antibodies against STC2 were used for pull-down from a mixture of the two proteins, followed by immunoblotting (Figure 1A; see Supplemental Figure 1 for uncropped blots). In parallel, a preparation of HRG serendipitously denatured during purification, was used as a negative control ("inactive HRG"). Active HRG was efficiently co-immunoprecipitated with STC2 while the inactive HRG was only inefficiently pulled down by STC2 (Figure 1B). Still, inactive HRG was detected by the polyclonal anti-HRG antibody upon immunoblotting, ensuring that this preparation indeed consisted of HRG. The inactive HRG-preparation was used as a negative control in subsequent experiments.

To demonstrate that the STC2 and HRG preparations were biologically active, their effects on the human histiocytic lymphoma cell line U937 was explored. U937 cells differentiate into

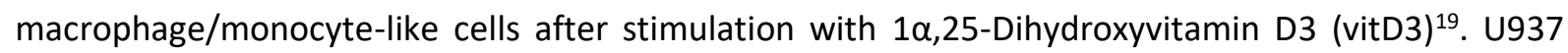
cells, vitD3-differentiated or not, were treated with HRG, STC2 or a mix of the two. Inactive HRG was tested in parallel. U937 cells were incubated with sterile bioparticles loaded with a $\mathrm{pH}$-sensitive fluorescent probe. Phagocytosis exposes the bioparticles to the low pH of the intracellular milieu, which leads to increased fluorescence. The change in fluorescence can be quantified as a measure of phagocytosis (Figure 1C and D). HRG, but not inactive HRG, increased the proportion of phagocytotic cells in both undifferentiated and differentiated cultures; however, in the undifferentiated cells, phagocytosis was 10 -fold lower than in the differentiated U937 monocytes. While STC2 alone lacked any effect on phagocytosis, it suppressed the increase in phagocytosis established in HRG-treated U937 monocytes (Figure 1D). These data demonstrate that the HRG and STC2 protein preparations are biologically active while the inactive HRG preparation lack effects.

We conclude that recombinant HRG and STC2 form a complex and that STC2 attenuates HRG's ability to upregulate phagocytosis by U937 monocytes.

\section{HRG and STC2 interact in a conformation-dependent manner}

HRG and STC2 are both secreted proteins, and therefore, it is unclear how they exert their modulatory effects on inflammatory cells. To investigate the interactions of the individual proteins and the complex 
127 with each other and with cells, we employed QCM technology ${ }^{21}$, allowing real-time and label-free evaluation of protein interactions in both a cell-free and a cellular environment.

129

First, STC2 was immobilized on the QCM chip surface and binding of STC2 to the surface was confirmed in the resulting sensorgram (Figure 2A). Next, binding of increasing concentrations of HRG to the STC2coated chip was analysed using a kinetic 1:1 global interaction model which showed an association rate $\left(\mathrm{K}_{\mathrm{a} 1}\right)$ of $2.6 \times 10^{4} \mathrm{M}^{-1} \cdot \mathrm{s}^{-1}$ and dissociation rate $\left(\mathrm{K}_{\mathrm{d} 1}\right)$ of $1.4 \times 10^{-3} \mathrm{~s}^{-1}$, resulting in a binding affinity of $55 \mathrm{nM}$ between HRG and STC2 (Figure 2B). Interestingly, in the inverse set-up, using HRG for immobilization, STC2 was not retained (Figure 2C). This result indicates that the interaction between HRG and STC2 is dependent on HRG's conformation which likely was compromised when immobilized onto the chip. In agreement, HRG is an intrinsically unstructured protein ${ }^{22}$, and therefore structurally less stable.

We conclude that HRG and STC2 bind to each other with relatively high affinity of $55 \mathrm{nM}$.

\section{Live U937 cells bind HRG after vitD3 differentiation}

Next, we assessed binding of HRG, STC2 and the complex to live U937 cells, vitD3-differentiated or not (Figure 3A). First, we confirmed the ability of U937 cells to differentiate to monocytes on the chip surface in response to vitD3. Relative CD14 expression increased $>1000$-fold after vitD3 treatment, ensuring that the cells indeed had differentiated to monocytes in response to vitD3 (Figure 3B). This is in agreement with previously reported effects of HRG on CD14 expression in vitD3-treated U937 cells ${ }^{13}$. Binding of both HRG and STC2 to undifferentiated U937 cells was weak (Figure 3C) but binding increased markedly when cells were differentiated (Figure 3D). The protein preparations, HRG or STC2 separately or mixed, bound to the U937 surface with similar properties, i.e. the HRG/STC2 complex did not show enhanced binding to cells (Figure 3D).

We conclude that both HRG and STC2 bound to U937 monocytes but not to undifferentiated cells and that the binding properties were very similar between the proteins, both when tested individually and as a complex.

\section{Binding of HRG to fixed U937 cells is independent of vitD3-induced differentiation}

Due to the challenge in separating interaction properties from interaction-induced changes in the live U937 monocytes immobilized on the QCM chip surface, cells were next fixed and binding of HRG and 
158

159

160

161

162

163

164

165

166

167

168

169

170

171

172

173

174

175

176

177

178

179

180

181

182

183

184

185

186

187

188

189

190

STC2 was determined. As expected, inactive HRG used as a negative control displayed no interaction with U937 cells, differentiated or not, even at high concentrations ( $100 \mu \mathrm{g} / \mathrm{ml}$ ) (Figure 4A). Also STC2 failed to bind both to undifferentiated and differentiated cells, with the response dropping down to baseline immediately after the end of injection (Figure 4B). In contrast, bioactive HRG interacted with the fixed cells with an affinity around $130 \mathrm{nM}$ to undifferentiated cells and $58 \mathrm{nM}$ to differentiated cells (Figure $4 \mathrm{C}$ and D). The results from the dissociation analysis showed 1:1 binding (Supplemental Figure $2 \mathrm{~A}$ and $\mathrm{B}$ ) and therefore a 1:1 interaction model was applied in the kinetic analysis. $\mathrm{K}_{\mathrm{a}}$ of $1.5 \mathrm{x}$ $10^{4} \mathrm{M}^{-1} \cdot \mathrm{s}^{-1}$ and $2.8 \times 10^{3} \mathrm{M}^{-1} \cdot \mathrm{s}^{-1}$ were recorded for the undifferentiated and vitD3-differentiated cells, while the $K_{d}$ was $2.0 \times 10^{-3} \cdot \mathrm{s}^{-1}$ and $1.5 \times 10^{-3} \cdot \mathrm{s}^{-1}$, respectively. Interestingly, the maximum binding response was slightly higher in the undifferentiated cells $(23.7 \mathrm{~Hz})$ compared to the differentiated cells (17.1 Hz).

Combined, these data show that fixation exposed binding sites for HRG on undifferentiated cells. We hypothesized that these binding sites may involve heparan sulfate epitopes ${ }^{6}$. To investigate this possibility further, cells were pre-incubated with heparinase before HRG binding. When the cells were treated with heparinase, a more sigmoidal dissociation curve was observed changing the interaction model to 1:2 interaction (Supplemental Figure $2 A$ and $B$ ). The $k_{a}$, when cells were treated with heparinase, was $7.9 \times 10^{3} \mathrm{M}^{-1} \cdot \mathrm{s}^{-1}$ and $2.4 \times 10^{4} \mathrm{M}^{-1} \cdot \mathrm{s}^{-1}$ for the undifferentiated and differentiated cells, respectively (Figure $4 \mathrm{E}$ and F). Moreover, $\mathrm{k}_{d}$ for undifferentiated, heparinase treated cells was $1.6 \times 10^{-}$ ${ }^{3} \cdot \mathrm{s}^{-1}$ and $1.1 \times 10^{-3} \cdot \mathrm{s}^{-1}$ for the differentiated, heparinase-treated cells. As expected, the Bmax was lower when the cells were treated with heparinase (Figure 4C, D, E and F). Interestingly, the heparinase treatment lowered the affinity of HRG to undifferentiated cells, from $130 \mathrm{nM}$ to $210 \mathrm{nM}$ resulting in an increased difference in affinity. In contrast, no difference in affinity was observed when the cells were differentiated, regardless of the treatment. This suggests that the heparan sulfate binding is more prominent in undifferentiated cells, and that heparinase treatment reveals other interaction surfaces for HRG, potentially a cell surface receptor, on differentiated cells. Moreover, the results support the assumption that the binding surface for STC2 and HRG are distinct and that the STC2 binding surface was denatured upon fixation of cells.

\section{Discussion}

This study aimed to understand the binding properties of the soluble plasma proteins HRG and STC2, motivated by their induction of gene regulatory programs that steer the phenotype of the monocyte/macrophage towards pro- or anti-inflammatory activities. We have previously shown that HRG, administered as recombinant protein, overexpressed by tumor cells, or delivered through 
191 adenovirus-mediated gene therapy, polarizes monocytes/macrophages to an anti-tumor immune 192 profile, allowing recruitment of cytotoxic T cells to the tumor ${ }^{1,14,23}$. The effect of HRG is accompanied 193 by tumor vessel normalization and suppressed tumor growth ${ }^{1,24}$. In a screen to identify HRG binding partners on the surface on monocytes mediating HRG's gene regulatory effects, STC2 was identified as a partner of HRG, with broad effects on inflammatory gene regulation ${ }^{14}$. Here, we asked whether HRG and STC2 steer monocyte gene regulation in a concerted action, based on the characteristics of binding to U937 monocytic cells.

To explore the binding properties of HRG and STC2, we employed QCM biosensor methodology using unmodified, label-free proteins. This is important as modifications such as fluorescent peptide linkers or fusion partners such as green fluorescent protein can affect folding of the modified protein and cause unnatural protein interactions. Moreover, radioactive labelling of proteins to determine protein interactions can harm the protein through the harsh methods used to introduce the label. In QCM, a thin quartz crystal disk is sandwiched between two electrodes. Changes in mass e.g. upon binding of HRG to the surface of immobilized cells, results in a mechanical deformation of the disk, which mediates a frequency change in a quartz crystal that is proportional to the change of mass, which allows for calculations of affinity ${ }^{25,26}$. The technology also allows to follow the kinetics of the interaction.

The main findings from this study are that HRG and STC2 bind to each other with nanomolar affinity and the interaction is stable as evidenced by the relatively slow dissociation rate. Both proteins also bind to differentiated, live U937 cells, i.e. to cells that in response to vitD3 have initiated a gene regulatory program similar to that accompanying monocyte differentiation, marked by expression of CD14 ${ }^{27}$. Individually, HRG and STC2 promote distinct U937 differentiation programs following vitD3 stimulation, with STC2 having more broad gene regulatory effects than $\mathrm{HRG}^{14}$. STC2 does not abrogate the effect of HRG on differentiation, but HRG dampens the gene regulatory effects of STC2 ${ }^{14}$. Still, STC2 suppressed the HRG-induced increase in phagocytosis in the U937 cells, demonstrating the multifaceted convergence of HRG/STC2-induced gene regulation. Overall, the data presented here indicate that HRG and STC2 bind to separate molecular entities on the surface of monocytes and that their gene regulatory effects may be exerted by convergence of downstream signaling pathways induced by each protein, rather than by the complex between the two.

We employed both live cells and cells fixed with formaldehyde for affinity studies. Utilization of fixed cells allows for repeated measurements and different concentrations of ligand on the same cell surfaces. This increases reproducibility and leads to more robust data. However, fixation may interfere 
preferred fixative for preserved immunoreactivity ${ }^{28}$. Upon fixation of cells, STC2 failed to bind to cells irrespective of the differentiation stage, while HRG bound to fixed cells both with and without vitD3 treatment (Figure 4). Therefore, we conclude that STC2 and HRG bind to distinct molecular entities on the U937 cells, and that the STC2 interactive surface was denatured upon fixation.

The binding of HRG to undifferentiated, fixed U937 cells may be due to fixation-induced exposure of heparan sulfate. HRG is known to bind heparan sulfate in a $\mathrm{Zn}^{2+}$-dependent manner, and this interaction is required for the anti-angiogenic effects of $\mathrm{HRG}^{6}$. We addressed the role of heparan sulfate by incubating cells with heparinase. Although incomplete, as revealed by the remaining binding of HRG to undifferentiated U937 cells, the digestion markedly changed the affinity of HRG for undifferentiated cells. Moreover, heparinase digestion resulted in a change of the interaction mode from a 1:1 model with linear dissociation curves to a 1:2 model with sigmoidal dissociation curves. This change supports the hypothesis that HRG binds two classes of binding epitopes with different affinities, heparan sulfate and a signal transducing receptor. Thus, treatment with heparinase decreases the binding of HRG to heparan sulfate and reveals the 1:2 binding interaction. These data underscore the relevance of using live cells, notwithstanding the associated challenges.

\section{Materials and methods}

\section{Differentiation of U937 cells}

The human histiocytic lymphoma cell line U93729 (American Type Culture Collection, ATCC 1593, RRID:CVCL_0007) was a kind gift from Prof Kenneth Nilsson, Uppsala University. The cells were cultured in RPMI 1640 medium supplemented with 10\% fetal bovine serum (FBS) and 1\% penicillin/streptomycin (Gibco 61870036). For monocyte differentiation, U937 cells were incubated in $10 \mathrm{nM}$ 1 $\alpha, 25$-Dihydroxyvitamin D3 (vitD3; Sigma 17936) for 15 hours, centrifuged (1500 rpm, 5 minutes), and resuspended in fresh medium. Differentiation was determined by real-time reverse transcriptase-PCR (qPCR) to detect CD14 transcripts. mRNA was extracted from cells using the RNAeasy mini kit (Qiagen) and RNA was reverse transcribed with iScript adv (cat no. 1725038, Bio-Rad). Gene expression was determined using TaqMan universal master mix (cat no. 4304437, Thermo Fisher) in the CFX96 Real-Time PCR Detection System (Bio-Rad) with TaqMan primers against human CD14 (cat no. Hs 00169122, Thermo Fisher) and human GAPDH (cat no. 4352934, Applied Biosystems). Cycle threshold values were calculated with CFX Maestro 1.1 software (Bio-Rad). . 
257

258

259

260

261

262

263

264

265

266

267

268

269

270

271

272

273

274

275

276

277

278

279

280

281

282

283

284

285

286

287

288

289

290

\section{Purified proteins and phagocytosis assay}

U937 cells were seeded at $10^{4}$ cells per well in 8-well chamber slides (ibidi 80826). At the start of the experiment, cells were incubated with $10 \mathrm{nM}$ vitD3, recombinant, in-house purified HRG (mouse) at 1 $\mu \mathrm{g} / \mathrm{ml}\left(13.3 \mathrm{nM}\right.$ ) ${ }^{23}$, STC2 (mouse) (cat no. STC2-16118M, Creative Biomart) or inactive HRG protein at equivalent molar concentrations together with sterile green E. coli bioparticles (cat. no. 4616, Essen Bioscience) at $33 \mu \mathrm{g} / \mathrm{ml}$. Following $20 \mathrm{~h}$ incubation at $37^{\circ} \mathrm{C}$ in $5 \% \mathrm{CO}_{2}$, cells were imaged at $10 \mathrm{X}$ with a Zeiss LSM 700 Microscope with AxioCam HRm and Zen software (Zeiss). Quantifications were done by automated counting of fluorescent cells in relation to all cells per image, using ImageJ (NIH).

\section{Co-immunoprecipitation and immunoblotting}

Equimolar concentrations of active or inactive HRG and STC2 were incubated on ice for 30 minutes followed by incubation with anti-STC2 antibody (Sigma hpa045372) for 1 hour. Protein G Sepharose (GE healthcare $71708300 \mathrm{AM}$ ) was as added and incubated at $4^{\circ} \mathrm{C}$ for 1 hour. Following centrifugation and washes, samples were heated at $97^{\circ} \mathrm{C}$ for 3 minutes for dissociation. Samples were separated by SDS-PAGE, transferred to PVDF membrane (Millipore), blocked in blocking buffer ( $5 \%$ milk in Trisbuffered saline and $0.1 \%$ Tween20) for 1 hour and incubated with primary HRG antibody (\#0119) ${ }^{7}$, overnight at $4^{\circ} \mathrm{C}$. Membranes were washed and incubated with HRP-conjugated secondary anti rabbit antibody (Thermo Fisher) in blocking buffer for 1 hour at room temperature. Development was performed with ECL prime (GE Healthcare) and luminescence signal detected using the ChemiDoc MP (Bio-Rad). Next, membranes were re-incubated with STC2 antibody (cat. no. hpa045372, Sigma) overnight at $4^{\circ} \mathrm{C}$ and developed again as described above.

\section{Binding of HRG to STC2}

Low noise block (LNB) chips were pre-wet with HEPES-buffered Steinberg's solution (HBS-T) and inserted in an Attana Cell ${ }^{\mathrm{TM}} 200$ instrument. When the signal was stabilized $(<0.2 \mathrm{~Hz})$, STC2 or HRG protein $(50 \mu \mathrm{g} / \mathrm{ml})$ were immobilized on the surface with a flow rate of $10 \mu \mathrm{l} / \mathrm{min}$ at $22^{\circ} \mathrm{C}$ using the amine coupling kit. Before running the biochemical assay, the signal was stabilized $(<0.2 \mathrm{~Hz})$. Different concentrations of HRG $(3.12,6.25$ and $12.5 \mu \mathrm{g} / \mathrm{mL}$ (each in triplicates) and STC2 $(7.5,15$ and $30 \mu \mathrm{g} / \mathrm{mL}$ ) were injected after blank injections (phosphate-buffered saline; PBS) followed by regeneration injections at $\mathrm{pH} 1$. PBS was used as a running buffer, for the blank injections and to dilute HRG. Glycine, $10 \mathrm{mM}, \mathrm{pH}$ 1, was used as regeneration buffer. The biochemical assay was carried out at a flow rate of $10 \mu \mathrm{l} / \mathrm{min}$, at $22^{\circ} \mathrm{C}$ and with $500 \mathrm{sec}$ of dissociation times. The data was prepared by subtracting the blank injections from the HRG injections using the Attana evaluation software. The curve fitting was performed using the Tracedrawer (Ridgeview Instruments), using the 
291

292

293

294

295

296

297

298

299

300

301

302

303

304

305

306

307

308

309

310

311

312

313

314

315

316

317

318

319

320

321

one-to-one and global model. The number of independent experiments (mostly 3 ) performed are given in the figure legends.

\section{Binding of HRG and STC2 to U937 cells treated or not with heparinase and fixative}

LNB-CC chips were pre-wet with HBS-T and then inserted in Attana Cell ${ }^{\mathrm{TM}} 200$. When the signal was stabilized $(<0.2 \mathrm{~Hz})$, lectin $(50 \mu \mathrm{g} / \mathrm{mL})$ was coupled by amine coupling. Cells were then seeded at a density of $2 \times 10^{5}$ cells per chip and left to settle for $45 \mathrm{~min}$ at room temperature. After seeding, cells were washed with PBS, stained with Hoechst 33342 solution for 15 minutes, and washed three times, followed by imaging using a fluorescence microscope (Nikon Eclipse 80i). Next, chips were inserted in the instrument Attana Cell ${ }^{\mathrm{TM}} 200$ ) and left to equilibrate $(<0.2 \mathrm{~Hz})$ under flow (RPMI 1640 medium, 20 $\mu \mathrm{l} / \mathrm{min}$ at $\left.37^{\circ} \mathrm{C}\right)$. STC2, HRG or a mix of the two $(10 \mu \mathrm{g} / \mathrm{ml})$ were injected manually over cells and responses were recorded for 30 minutes. When indicated, cells were treated with heparinase using a mixture of heparinase-I, -II, and -III (IBEX Pharmaceuticals), which was added to the cultures at a final concentration of $3.4 \mathrm{mU} / \mathrm{ml}$ for each enzyme, for $1 \mathrm{~h}$ at $37^{\circ} \mathrm{C}$ before seeding of cells on the activated chips. The fixation of cells was performed just after seeding by removing the PBS and adding $50 \mu \mathrm{L}$ of $3.7 \%$ formaldehyde solution. Subsequently, the chips were incubated at $4^{\circ} \mathrm{C}$ for 10 minutes followed by washing with PBS three times. The data was prepared by subtracting the blank injections from the analyte injections using the Attana evaluation software. The curve fitting was performed using the Tracedrawer (Ridgeview Instruments), using the one-to-one or one-to-two binding models (only one component reported) and global model. At least two independent experiments were performed.

\section{Acknowledgements}

This study was supported by the Swedish Cancer foundation (19 0119 Pj $01 \mathrm{H}$ ), the Swedish Research Council (2020-01349), the Knut and Alice Wallenberg foundation (project grant KAW 2020.0057 and Wallenberg Scholar grant (2015.0275).

\section{Author contributions}

Conceptualization: TPS, HK, LCW. Investigation: TSP, CD. Methodology development: CD, TA. Evaluation: All authors. Statistics: TSP, CD, TA. Writing original draft: TSP, LCW. Editing: All authors. Financing: LCW. 


\section{Disclosures}

TSP, HK, LCW declare no financial interest. CD is an employee of and TA is the founder and CEO of Attana Ab.

\section{References}

1 Rolny, C. et al. HRG inhibits tumor growth and metastasis by inducing macrophage polarization and vessel normalization through downregulation of PIGF. Cancer Cell 19, 31-44, doi:10.1016/j.ccr.2010.11.009 (2011).

2 Wakabayashi, S. in International Review of Cell and Molecular Biology Vol. 304 (ed Kwang W. Jeon) 467-493 (Academic Press, 2013).

3 Poon, I. K., Patel, K. K., Davis, D. S., Parish, C. R. \& Hulett, M. D. Histidine-rich glycoprotein: the Swiss Army knife of mammalian plasma. Blood 117, 2093-2101, doi:10.1182/blood-2010-09303842 (2011).

4 Shannon, O. et al. Histidine-rich glycoprotein promotes bacterial entrapment in clots and decreases mortality in a mouse model of sepsis. Blood 116, 2365-2372, doi:10.1182/blood2010-02-271858 (2010).

5 Gorgani, N. N., Parish, C. R., Easterbrook Smith, S. B. \& Altin, J. G. Histidine-Rich Glycoprotein Binds to Human IgG and C1q and Inhibits the Formation of Insoluble Immune Complexes. Biochemistry 36, 6653-6662, doi:10.1021/bi962573n (1997).

6 Vanwildemeersch, M. et al. The anti-angiogenic His/Pro-rich fragment of histidine-rich glycoprotein binds to endothelial cell heparan sulfate in a $\mathrm{Zn2+-dependent} \mathrm{manner.} \mathrm{The}$ Journal of biological chemistry 281, 10298-10304, doi:10.1074/jbc.M508483200 (2006).

7 Olsson, A.-K. et al. A fragment of histidine-rich glycoprotein is a potent inhibitor of tumor vascularization. Cancer research 64, 599-605 (2004).

8 Blank, M. \& Shoenfeld, Y. Histidine-rich glycoprotein modulation of immune/autoimmune, vascular, and coagulation systems. Clinical reviews in allergy \& immunology 34, 307-312, doi:10.1007/s12016-007-8058-6 (2008).

9 Roche, F. et al. Histidine-rich glycoprotein blocks collagen-binding integrins and adhesion of endothelial cells through low-affinity interaction with alpha2 integrin. Matrix Biol 48, 89-99, doi:10.1016/j.matbio.2015.06.002 (2015).

10 Kuroda, K. et al. Histidine-rich glycoprotein as a prognostic biomarker for sepsis. Scientific reports 11, 10223, doi:10.1038/s41598-021-89555-z (2021). 
35511 Tsuchida-Straeten, N. et al. Enhanced blood coagulation and fibrinolysis in mice lacking 356 histidine-rich glycoprotein (HRG). Journal of thrombosis and haemostasis : JTH 3, 865-872, doi:10.1111/j.1538-7836.2005.01238.x (2005).

12 Lee, C., Bongcam-Rudloff, E., Sollner, C., Jahnen-Dechent, W. \& Claesson-Welsh, L. Type 3 cystatins; fetuins, kininogen and histidine-rich glycoprotein. Frontiers in bioscience (Landmark edition) 14, 2911-2922, doi:10.2741/3422 (2009).

13 Tugues, S. et al. Genetic deficiency in plasma protein HRG enhances tumor growth and metastasis by exacerbating immune escape and vessel abnormalization. Cancer Res 72, 19531963, doi:10.1158/0008-5472.Can-11-2194 (2012).

14 Roche, F. P. et al. Leukocyte Differentiation by Histidine-Rich Glycoprotein/Stanniocalcin-2 Complex Regulates Murine Glioma Growth through Modulation of Antitumor Immunity. Mol Cancer Ther 17, 1961-1972, doi:10.1158/1535-7163.MCT-18-0097 (2018).

15 Uhlén, M. et al. Proteomics. Tissue-based map of the human proteome. Science (New York, N.Y.) 347, 1260419, doi:10.1126/science.1260419 (2015).

16 Joshi, A. D. New Insights Into Physiological and Pathophysiological Functions of Stanniocalcin

17 Yeung, B. H., Law, A. Y. \& Wong, C. K. Evolution and roles of stanniocalcin. Molecular and cellular endocrinology 349, 272-280, doi:10.1016/j.mce.2011.11.007 (2012).

18 Chang, A. C. et al. The murine stanniocalcin 2 gene is a negative regulator of postnatal growth. Endocrinology 149, 2403-2410, doi:10.1210/en.2007-1219 (2008).

19 Olsson, I., Gullberg, U., Ivhed, I. \& Nilsson, K. Induction of differentiation of the human histiocytic lymphoma cell line U-937 by 1 alpha,25-dihydroxycholecalciferol. Cancer Res 43, 5862-5867 (1983).

Gianneli, M. et al. Label-free in-flow detection of receptor recognition motifs on the biomolecular corona of nanoparticles. Nanoscale 10, 5474-5481, doi:10.1039/c7nr07887k (2018).

1 Peiris, D. et al. in Epitope Mapping Protocols (eds Johan Rockberg \& Johan Nilvebrant) 53-63 (Springer New York, 2018).

22 Tompa, P. Intrinsically unstructured proteins. Trends in biochemical sciences 27, 527-533, doi:10.1016/s0968-0004(02)02169-2 (2002).

23 Tugues, S. et al. Histidine-rich glycoprotein uptake and turnover is mediated by mononuclear phagocytes. PLoS One 9, e107483, doi:10.1371/journal.pone.0107483 (2014).

24 Theek, B. et al. Histidine-rich glycoprotein-induced vascular normalization improves EPRmediated drug targeting to and into tumors. Journal of controlled release : official journal of the Controlled Release Society 282, 25-34, doi:10.1016/j.jconrel.2018.05.002 (2018). 
25 Marx, K. A. Quartz Crystal Microbalance: A Useful Tool for Studying Thin Polymer Films and Complex Biomolecular Systems at the Solution-Surface Interface. Biomacromolecules 4, 10991120, doi:10.1021/bm020116i (2003).

Pei, Z., Saint-Guirons, J., Käck, C., Ingemarsson, B. \& Aastrup, T. Real-time analysis of the carbohydrates on cell surfaces using a QCM biosensor: a lectin-based approach. Biosensors \& bioelectronics 35, 200-205, doi:10.1016/j.bios.2012.02.047 (2012). Zamani, F., Zare Shahneh, F., Aghebati-Maleki, L. \& Baradaran, B. Induction of CD14 Expression and Differentiation to Monocytes or Mature Macrophages in Promyelocytic Cell Lines: New Approach. Advanced pharmaceutical bulletin 3, 329-332, doi:10.5681/apb.2013.053 (2013). Paavilainen, L. et al. The impact of tissue fixatives on morphology and antibody-based protein profiling in tissues and cells. The journal of histochemistry and cytochemistry : official journal of the Histochemistry Society 58, 237-246, doi:10.1369/jhc.2009.954321 (2010). Sundström, lymphoma cell line (U-937)

ablishment and d characterization of a human histiocytic 403 doi:10.1002/ijc.2910170504 (1976).

405 


\section{Figure legends}

Figure 1 Recombinant HRG binds STC2 and modulates phagocytosis of bioparticles

A. Co-immunopreciptation of HRG but not inactive HRG with STC2. STC2, HRG and inactive HRG (2 $\mu \mathrm{g}$ each) were separated on SDS-PAGE as individual preparations (loading control) or after mixing and immunoprecipitation (IP) using antibodies against STC2, followed by immunoblotting (IB) as indicated.

B. Ratio of HRG (active or inactive) band intensities in the STC2 immunoprecipitate normalized to corresponding active and inactive HRG loading controls.

C. Representative microscope images of U937 monocytes without (left) or with treatment with active HRG (right) in the phagocytosis assay. Green cells have engulfed pH-sensitive fluorescent bioparticles.

D. Quantification of phagocytosis efficiency in the different treatment conditions. The proportion of positive (green) phagocytotic U937 cells to all cells per field of vision is shown in relation to the positive cells/total cells in vitD3 differentiated HRG-treated condition (set to 1). Statistical analysis with Students T-test (B) and Tukey's multiple comparisons test (D). $P<0.05$ considered significant. Scale bar in C; $50 \mu \mathrm{m}$.

\section{Figure 2. Affinity determination of HRG's binding to STC2 using QCM}

A. Immobilization of STC2 on the QCM LNB sensor surface

B. Sensorgrams and kinetic analysis showing chip-immobilized STC2 and binding of HRG at three different concentrations; 50, 100 and 200 nM. Black lines: experimental curves. Red lines: fitted curves. Representative sensorgram shown, three injections per concentration, two independent experiments.

C. Sensorgrams showing chip-immobilized HRG and lack of binding of STC2, tested th three different concentrations; 200 nM, $450 \mathrm{nM}$ and $900 \mathrm{nM}$. Representative sensorgram shown, two injections per concentration, three independent experiments.

Figure 3. Binding of HRG and STC2 individually and together to live U937 cells

A. Schematic of experimental setup. Undifferentiated or vitD3 differentiated U937 cells, immobilized on QCM LNB chips with HRG, STC2 or a mix of the two, injected over chip surfaces.

B. Real-time qPCR data of CD14 expression normalized to GAPDH, on undifferentiated and vitD3 differentiated U937 cells seeded on the QCM chip. 
436 C, D. Representative sensorgram showing frequency response from injections over undifferentiated

437 (C) and vitD3 differentiated (D) U937 cells. Representative sensorgram shown, two independent 438 experiments.

439

440 Figure 4. Affinity determination of HRG to fixed U937 cells

441 A. Sensorgram showing frequency response to inactive HRG over vitD3 differentiated, fixed U937 cells.

442 B. Sensorgrams showing frequency response to four concentrations ( $125 \mathrm{nM}, 250 \mathrm{nM}, 500 \mathrm{nM}, 1 \mu \mathrm{M}$ )

443 of STC2 over fixed, undifferentiated (dashed lines) and vitD3 differentiated (straight lines) U937 cells.

444 Mean of two injections shown.

445 C, D. Sensorgrams and kinetic analysis showing binding of $H R G$ at three concentrations $(25,50$ and 100

$446 \mathrm{nM}$ ) to undifferentiated (C) or vitD3 differentiated (D), fixed U937 cells. Black lines: experimental

447 curves. Red lines: fitted curves. Representative sensorgrams shown, three injections per

448 concentration, three independent experiments.

449 E, F. Sensorgrams and kinetic analysis showing frequency response to three concentrations of HRG (25, 45050 and $100 \mathrm{nM}$ ) to fixed, undifferentiated (E) or vitD3 differentiated (F) U937 cells, after treatment 451 with heparinase. Black lines: experimental curves. Red lines: fitted curves. Means of three independent 452 experiments shown, three injections per concentration, three independent experiments.

453

454 


\section{Figures}

A

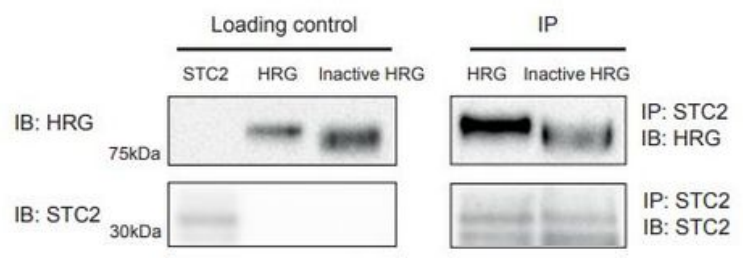

C

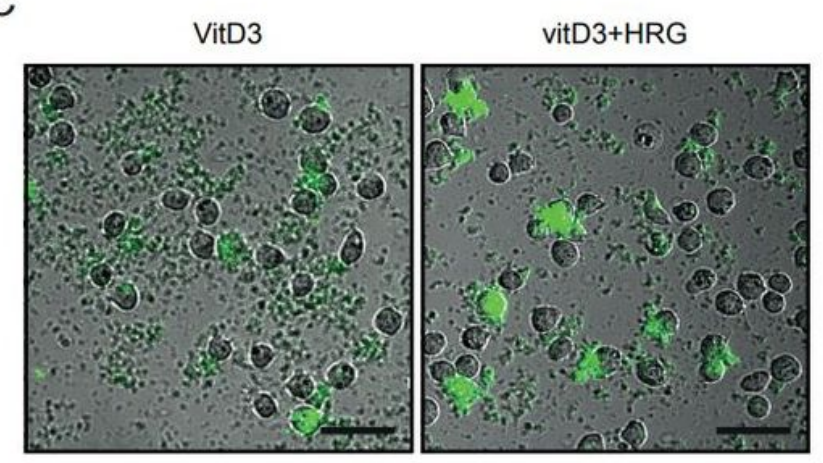

B
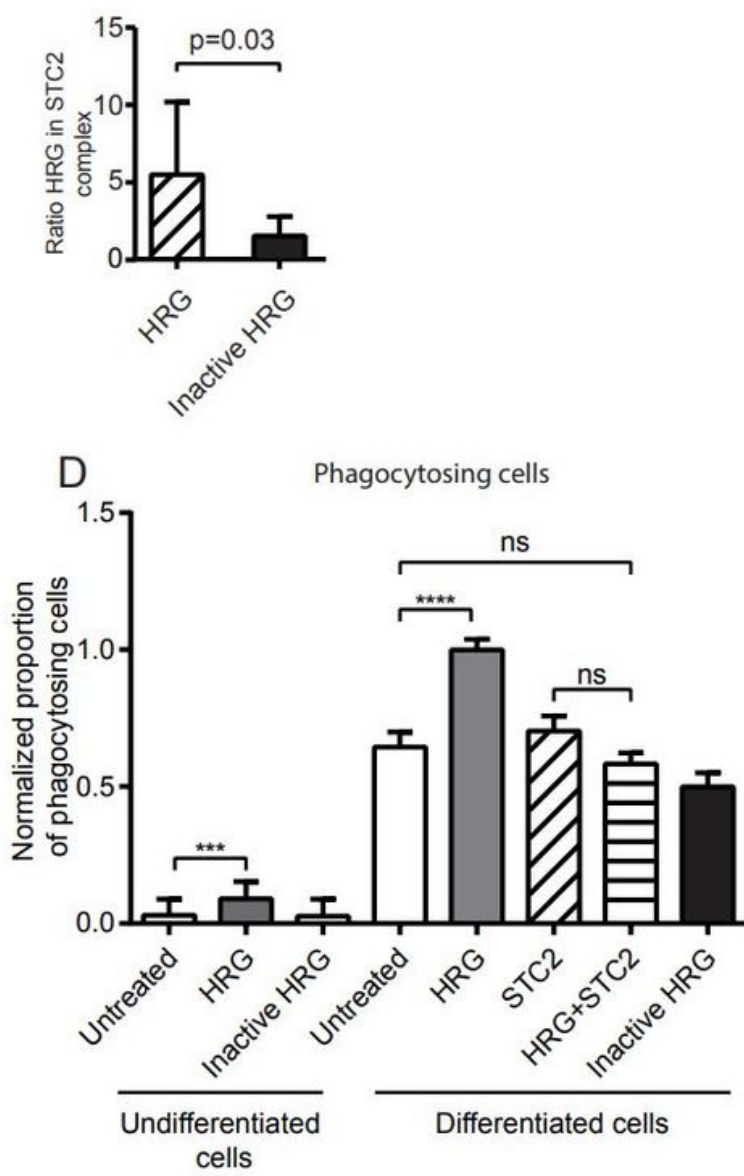

\section{Figure 1}

Recombinant HRG binds STC2 and modulates phagocytosis of bioparticles

A. Co-immunopreciptation of HRG but not inactive HRG with STC2. STC2, HRG and inactive HRG ( $2 \mu \mathrm{g}$ each) were separated on SDS-PAGE as individual preparations (loading control) or after mixing and immunoprecipitation (IP) using antibodies against STC2, followed by immunoblotting (IB) as indicated.

B. Ratio of HRG (active or inactive) band intensities in the STC2 immunoprecipitate normalized to corresponding active and inactive HRG loading controls.

C. Representative microscope images of U937 monocytes without (left) or with treatment with active HRG (right) in the phagocytosis assay. Green cells have engulfed pH-sensitive fluorescent bioparticles.

D. Quantification of phagocytosis efficiency in the different treatment conditions. The proportion of positive (green) phagocytotic U937 cells to all cells per field of vision is shown in relation to the positive 
cells/total cells in vitD3 differentiated HRG-treated condition (set to 1). Statistical analysis with Students T-test (B) and Tukey's multiple comparisons test (D). P< 0.05 considered significant. Scale bar in C; 50 $\mu \mathrm{m}$.

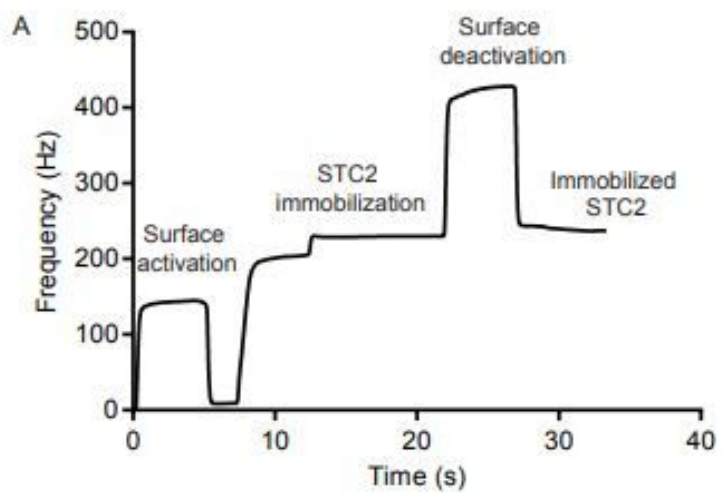

B

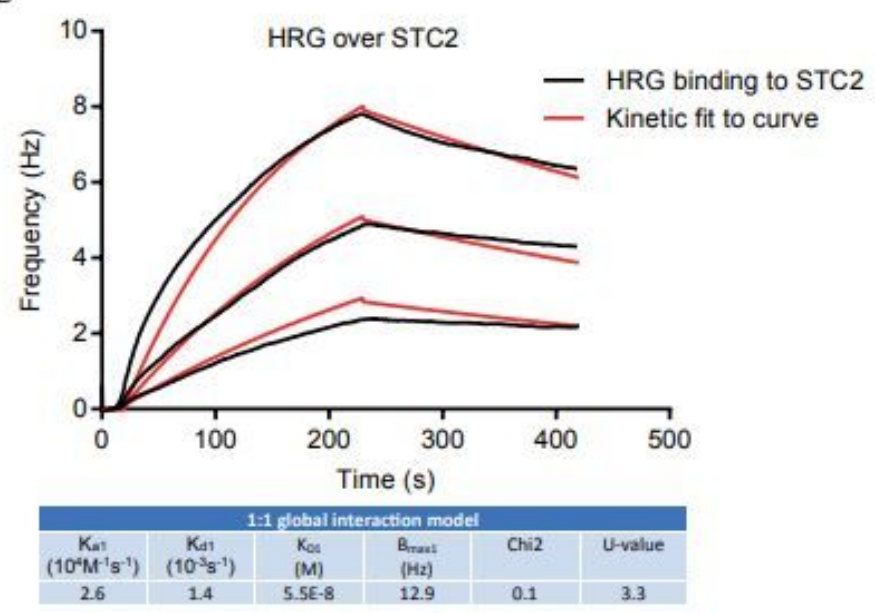

C

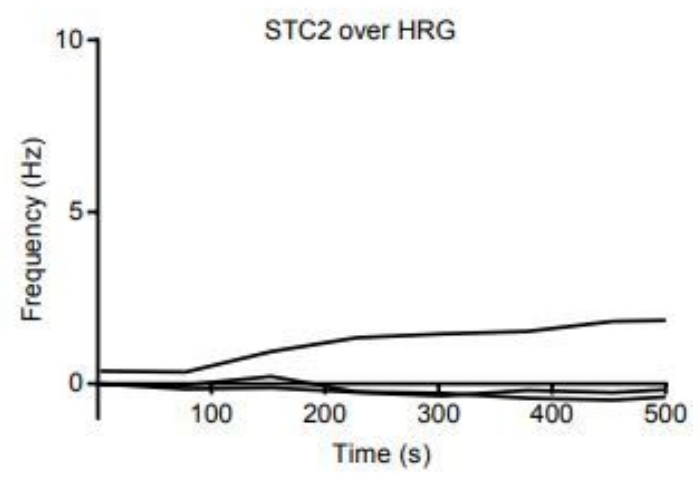

Figure 2 
A. Immobilization of STC2 on the QCM LNB sensor surface

B. Sensorgrams and kinetic analysis showing chip-immobilized STC2 and binding of HRG at three different concentrations; 50, 100 and 200 nM. Black lines: experimental curves. Red lines: fitted curves. Representative sensorgram shown, three injections per concentration, two independent experiments.

C. Sensorgrams showing chip-immobilized HRG and lack of binding of STC2, tested at three different concentrations; 200 nM, 450 nM and 900 nM. Representative sensorgram shown, two injections per concentration, three independent experiments.

A
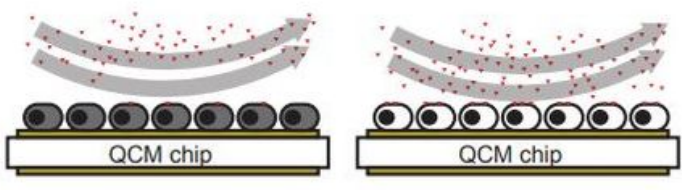

(2) Undifferentiated U937 cell

D Differentiated U937 cell

- Proteins

C

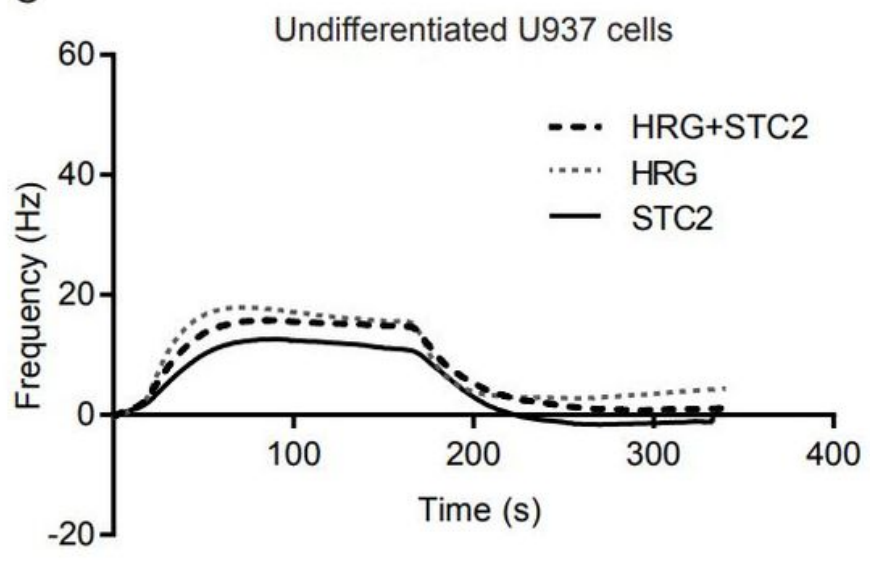

B

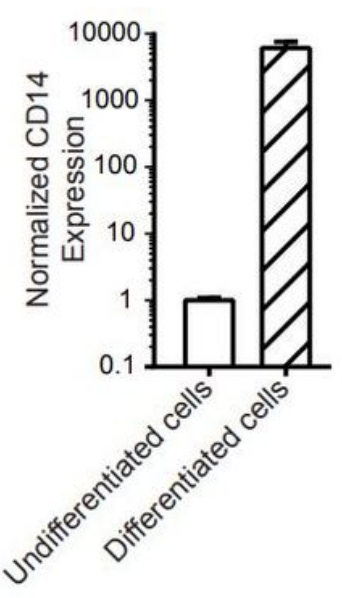

D

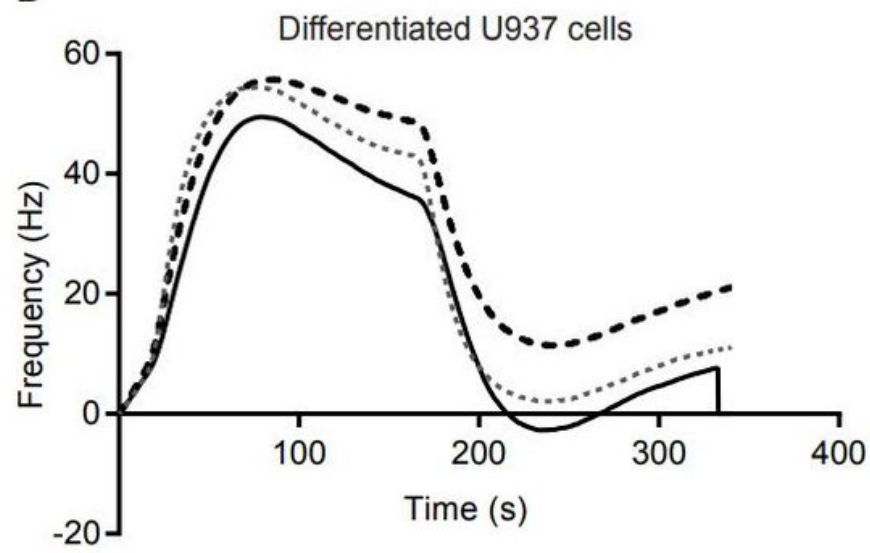

\section{Figure 3}

Binding of HRG and STC2 individually and together to live U937 cells

A. Schematic of experimental setup. Undifferentiated or vitD3 differentiated U937 cells, immobilized on QCM LNB chips with HRG, STC2 or a mix of the two, injected over chip surfaces.

B. Real-time qPCR data of CD14 expression normalized to GAPDH, on undifferentiated and vitD3 differentiated U937 cells seeded on the QCM chip. 
C, D. Representative sensorgram showing frequency response from injections over undifferentiated (C) and vitD3 differentiated (D) U937 cells. Representative sensorgram shown, two independent experiments.

A

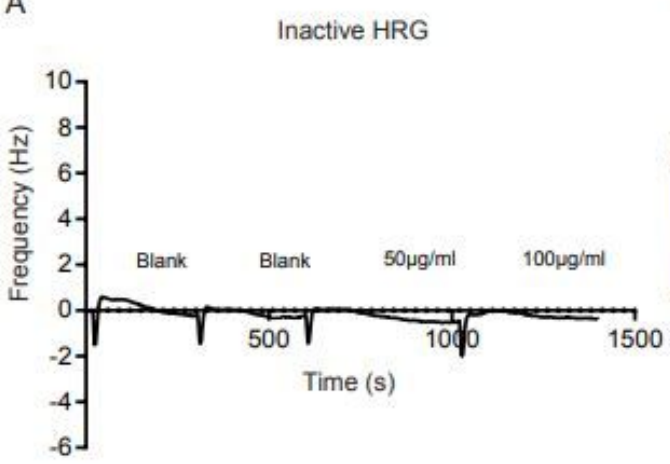

C

HRG on undifferentiated, fixed U937
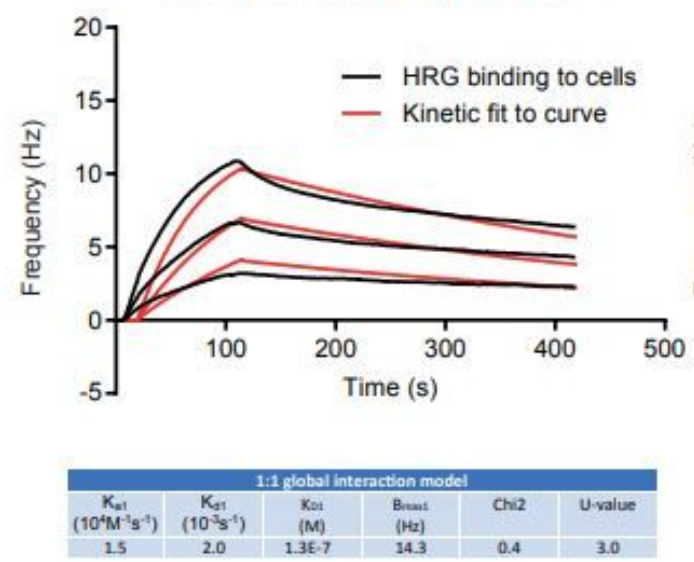

E

HRG + heparinase on undifferentiated, fixed U937

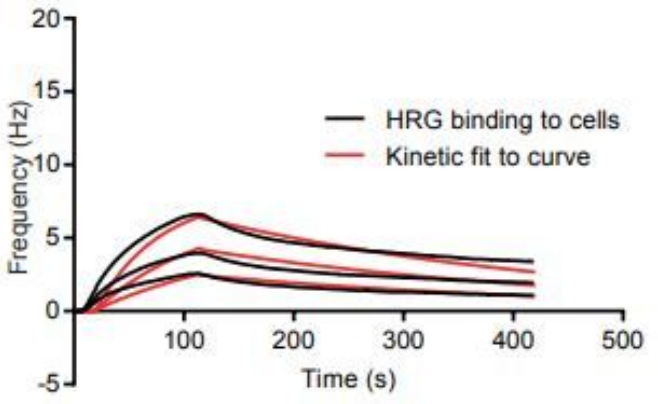

\begin{tabular}{|c|c|c|c|c|c|}
\hline \multicolumn{6}{|c|}{ 1:2 global Internction model } \\
\hline $\begin{array}{c}K_{\text {-1 }} \\
\left(10^{-1} M^{-1-1} s^{-4}\right)\end{array}$ & $\begin{array}{c}K_{\mathrm{di}} \\
\left(10^{-1-1} \mathrm{~s}^{-1}\right)\end{array}$ & $\begin{array}{l}K_{01} \\
\text { (M) }\end{array}$ & $\begin{array}{l}\mathrm{B}_{\operatorname{mat}} \\
\langle\mathrm{Hz}\rangle\end{array}$ & $\mathrm{Ch}$ : & U-value \\
\hline 7.9 & 1.6 & 2.1E:7 & 10.8 & 0.03 & 8.6 \\
\hline $\begin{array}{c}K_{w a} \\
\left(10^{2} M^{-1} s^{-1}\right)\end{array}$ & $\begin{array}{c}K_{12} \\
\left(10^{-2} s^{-1}\right)\end{array}$ & $\begin{array}{l}K_{\infty 00} \\
(\mathrm{M})\end{array}$ & $\begin{array}{l}\mathrm{B}_{\ln 20} \\
(\mathrm{~Hz})\end{array}$ & Chiz & U-value \\
\hline 1.9 & 4.6 & 2.5E.7 & 20 & 0.03 & 8.6 \\
\hline
\end{tabular}

B

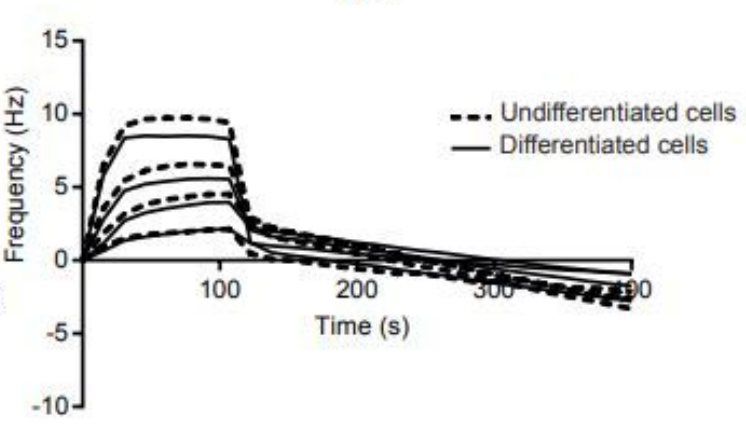

D

HRG on differentiated, fixed U937
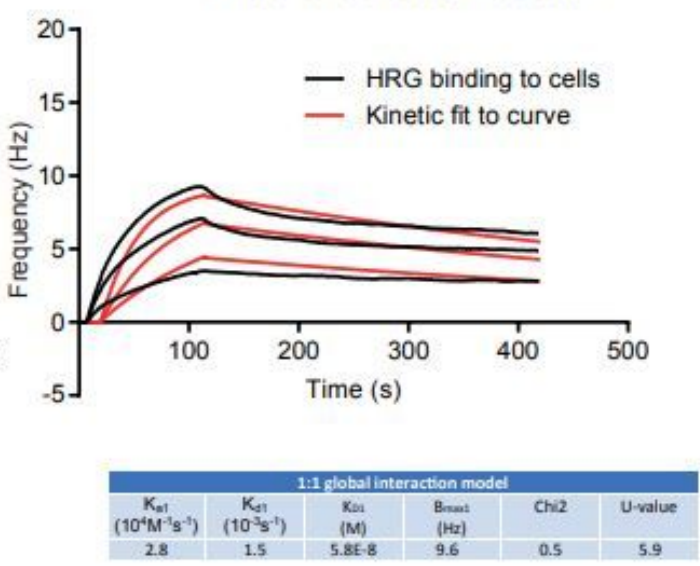

F

HRG + heparinase on differentiated, fixed U937

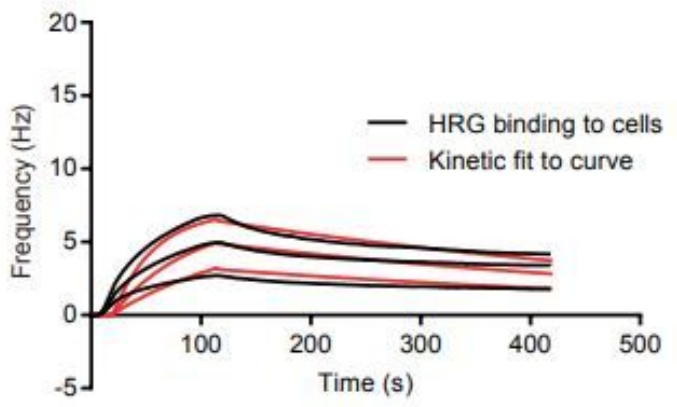

\begin{tabular}{|c|c|c|c|c|c|}
\hline \multicolumn{6}{|c|}{$1: 2$ slobal Interaction model } \\
\hline $\begin{array}{c}K_{\text {m1 }} \\
\left(10^{4} M^{-1-1} s^{-1}\right)\end{array}$ & $\begin{array}{c}K_{11} \\
\left(10^{-1} s^{-1}\right)\end{array}$ & $\begin{array}{l}\mathrm{K}_{\text {in }} \\
\text { (M) }\end{array}$ & $\begin{array}{l}B_{\operatorname{man}} \\
\langle\mathrm{Hz}\rangle\end{array}$ & $\mathrm{Ch} / 2$ & U-value \\
\hline 2.4 & 1.1 & $5.2 E-8$ & 7.0 & 0.1 & 24.4 \\
\hline $\begin{array}{c}K_{2 z} \\
\left(10^{5} M^{-1-1} s^{-1}\right)\end{array}$ & $\begin{array}{c}K_{a n} \\
\left(10^{22} s^{-1}\right)\end{array}$ & $\begin{array}{l}K_{\infty 00} \\
(M)\end{array}$ & $\begin{array}{l}\mathrm{B}_{\text {nam }} \\
(\mathrm{Hz})\end{array}$ & $\mathrm{Ch} / 2$ & U-value \\
\hline 3.1 & 7.6 & 2.56 .7 & 2.0 & 0.1 & 24.4 \\
\hline
\end{tabular}

Figure 4

Affinity determination of HRG to fixed U937 cells 
A. Sensorgram showing frequency response to inactive HRG over vitD3 differentiated, fixed U937 cells.

B. Sensorgrams showing frequency response to four concentrations $(125 \mathrm{nM}, 250 \mathrm{nM}, 500 \mathrm{nM}, 1 \mu \mathrm{M})$ of STC2 over fixed, undifferentiated (dashed lines) and vitD3 differentiated (straight lines) U937 cells. Mean of two injections shown.

C, D. Sensorgrams and kinetic analysis showing binding of HRG at three concentrations $(25,50$ and 100 $\mathrm{nM}$ ) to undifferentiated (C) or vitD3 differentiated (D), fixed U937 cells. Black lines: experimental curves. Red lines: fitted curves. Representative sensorgrams shown, three injections per concentration, three independent experiments.

E, F. Sensorgrams and kinetic analysis showing frequency response to three concentrations of HRG (25, 50 and $100 \mathrm{nM}$ ) to fixed, undifferentiated (E) or vitD3 differentiated (F) U937 cells, after treatment with heparinase. Black lines: experimental curves. Red lines: fitted curves. Means of three independent experiments shown, three injections per concentration, three independent experiments.

\section{Supplementary Files}

This is a list of supplementary files associated with this preprint. Click to download.

- SupplementaryInformationPerssonSkare.pdf 